\title{
Problem of data interpretation of laser polarization sensing of high-level clouds on the basis of theoretically calculated phase matrices of backscattering by monodisperse ice crystals
}

\author{
Ignatii Samokhvalov * and Ilia Bryukhanov \\ National Research Tomsk State University, Tomsk, Russia 634050
}

\begin{abstract}
A technique for determining the microstructure parameters of high-level clouds on the basis of joint analysis of experimental and theoretical data is described. Results of the experiments on atmosphere laser sensing and theoretical calculations of light scattering by spatially oriented ice crystals are shown.
\end{abstract}

\section{Introduction}

There is an increasing emphasis on investigations of radiative properties of high-level clouds (HLC) related with their important role in the formation of the Earth's climate and radiation budget. The lack of consideration of accounting the effect of a number of phenomena and processes occurring in these clouds leads to inaccuracies in the calculations of the radiation budget. Spatial orientation of non-spherical ice crystals in clouds is one of such poorly known factors.

The effect of particles in clouds on the transmission of incoming solar radiation and Earth's outgoing thermal radiation determines the need of creating a microphysical model of HLCs considering this parameter. The absence of such models is a consequence of the impossibility of measuring the orientation of aerosol particles in clouds by contact methods because of its violation during sampling, for example using an aircraft.

\section{Determination of backscattering phase matrices and experimental data processing}

Full information on a cloud microstructure is contained in its backscattering phase matrix (BSPM), which relates polarization characteristics of sensing laser radiation and radiation backscattered by cloud particles. Thus, to evaluate the microstructure parameters of the cloud, it is necessary to determine its BSPM. To investigate special features of cirrus clouds, polarization laser sensing method [1] is used in the high-altitude polarization lidar developer at National Research Tomsk State University (TSU; Tomsk, Russia) [2]. Transmission system of the lidar sends radiation with 4 various polarization states, which

*Corresponding author: lidar@mail.tsu.ru 
are described by the Stokes vector $\boldsymbol{S}_{\boldsymbol{n}}$ ( $n$ takes values from 1 to 4 ) into the atmosphere. Receiving system of the lidar implements consequently 4 various instrument vectors $\boldsymbol{G}_{\boldsymbol{m}}(m$ takes values from 1 to 4 ) that provides determining the polarization state of backscattered radiation. Unlike existing analogues [for example 3-5], the use of original technical solutions in the lidar developed at TSU allows all 16 elements of a BSPM to be determined experimentally without the use of the properties of pairwise equality of its elements. Wherein, single scattering approximation is used, as it is argued in [6]. Results of the experiments on polarization laser sensing of the atmosphere are compared with meteorological parameters at the altitudes of HLC formation [2]. A schematic diagram of the lidar is shown in Fig. 1.

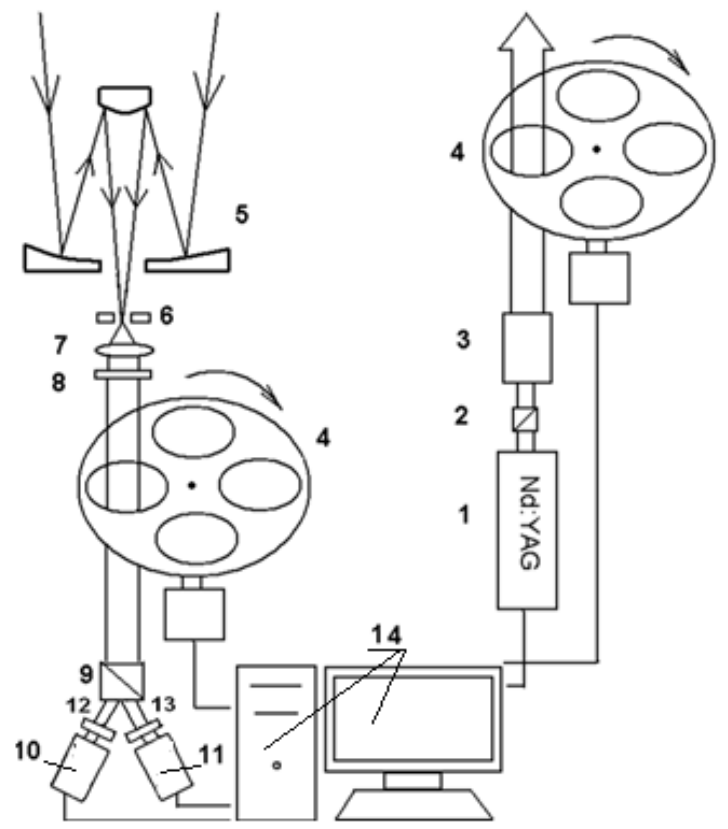

Fig. 1. Schematic diagram of the polarization lidar developed at TSU: 1 is a Nd:YAG laser $(\lambda=532 \mathrm{~nm}) ; 2$ is a Glan prism; 3 is a collimator; 4 are units that transform the polarization state of transmitting and receiving radiation; 5 is a Cassegrain mirror objective; 6 is a field stop; 7 is a lens; 8 is an interference filter; 9 is a Wollaston prism; 10 and 11 are photomultiplier tubes; 12 and 13 are electrooptical electrooptical shutters; and 14 is a computer-based equipment for registration and displaying the data [2].

The lidar is built on a monostatic scheme with spaced optical axes of the receiving and transmitting antennas. A Nd:YAG laser with a working wavelength of $532 \mathrm{~nm}$, pulse repetition frequency of $10 \mathrm{~Hz}$, and energy per pulse of up to $400 \mathrm{~mJ}$ was used as a source of sensing radiation. The Cassegrain telescope with a diameter of the main mirror of $0.5 \mathrm{~m}$ and a focal distance of $5 \mathrm{~m}$ served as a receiving objective. A field stop specifying the field-ofview angle of the receiving system, a positive lens transforming a diverging beam into a quasi-parallel one, an interference filter, and a Wollaston prism, at the exit from which two beams were formed with mutually orthogonal polarization states, were successively arranged in the receiving channel along the propagation path of scattered radiation. The beam intensities were registered with Hamamatsu photomultipliers (PMTs); the registration system includes a single-electron pulse counter. Two optoelectrooptical shutters, which suppress backscattering interferences from the lidar near zone, were mounted before the PMTs. System for collection, processing, and displaying the experiemntal data is based on a computer. Lidar is controlled by an operator in semi-automatic mode. 
Sensing is performed in the zenith direction. Parallel and orthogonal polarization components of backscattered radiation are measured simultaneously. Both these facts provide the detection of ensembles of aerosol particles with preferred horizontal spatial orientation [7]. One of the special features of such orientation in polarization laser sensing is a low depolarization of sensing radiation upon its interacting with ice crystals oriented horizontally. The presence of a useful signal in one of the polarization channels of the lidar receiving system and its absence in another one during measurements that use combinations of the same polarization elements in the lidar transmitter and receiver allows this situation to be identified. Moreover, a number of special features of such mirror clouds are revealed on the basis of the analysis of the experimental data obtained for all operation time of the lidar. Such clouds are formed at the altitudes from 8 to $12 \mathrm{~km}$, have the geometric thickness of not more than $1.5 \mathrm{~km}$, anomalous high scattering ratio $20<R(h)<200$, and small optical thickness $\tau<1$ [2].

Figure 2 shows the examples of the experimental data. Each vertical profile of the lidar signal power is obtained using an accumulation of 500 sensing pulses with a gate length of $75 \mathrm{~m}$. There were two cloud layers: at the altitudes of $7.7-9.5$ and $10.6-11.7 \mathrm{~km}$, respectively.
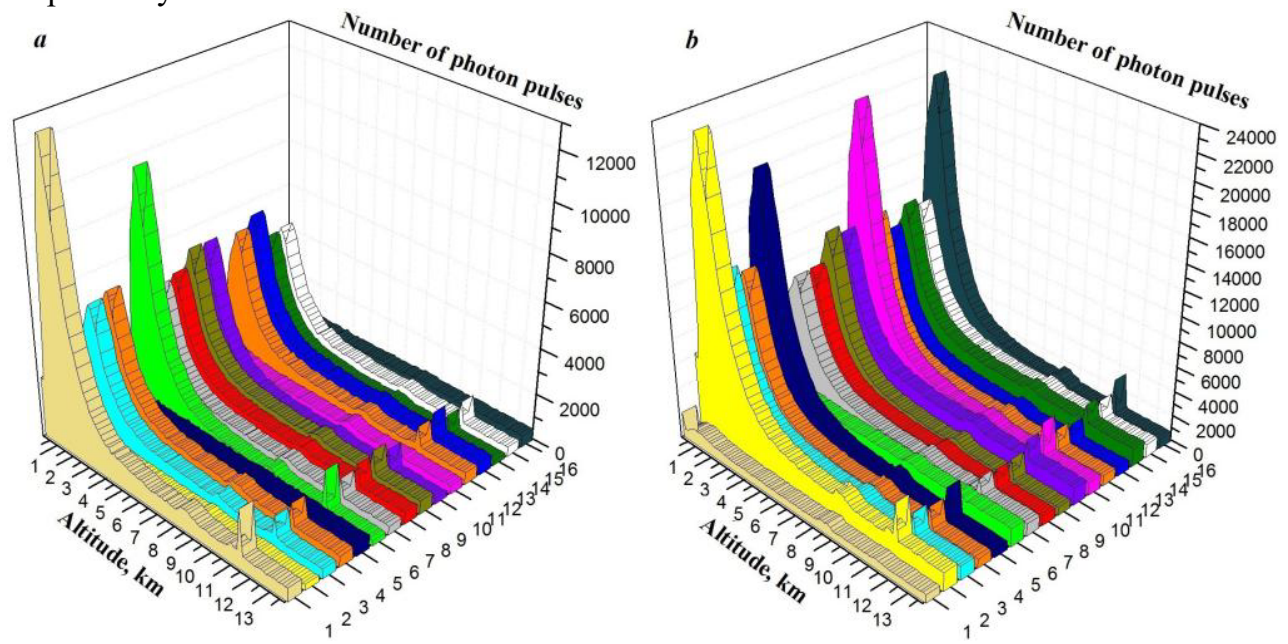

$\square$ (1) $\mathrm{S}=\left(\begin{array}{llll}1 & 1 & 0 & 0\end{array}\right)^{\mathrm{T}}, \mathrm{G}=\left(\begin{array}{lll}1 & 1 & 0\end{array}\right) / 2 \square(5) \mathrm{S}=\left(\begin{array}{llll}1 & -1 & 0 & 0\end{array}\right)^{\mathrm{T}}, \mathrm{G}=\left(\begin{array}{llll}1 & 1 & 0 & 0\end{array}\right) / 2$

(2) $\mathrm{S}=\left(\begin{array}{llll}1 & 1 & 0 & 0\end{array}\right)^{\mathrm{T}}, \mathrm{G}=\left(\begin{array}{llll}1 & -1 & 0 & 0\end{array}\right) / 2 \square(6) \mathrm{S}=\left(\begin{array}{llll}1 & -1 & 0 & 0\end{array}\right)^{\mathrm{T}}, \mathrm{G}=\left(\begin{array}{llll}1 & -1 & 0 & 0\end{array}\right) / 2$

(3) $\mathrm{S}=\left(\begin{array}{llll}1 & 1 & 0 & 0\end{array}\right)^{\mathrm{T}}, \mathrm{G}=\left(\begin{array}{llll}1 & 0 & 0 & -1\end{array}\right) / 2 \square\left(\begin{array}{lll}7 \\ \mathrm{C}\end{array}\right) \mathrm{S}=\left(\begin{array}{llll}1 & -1 & 0 & 0\end{array}\right)^{\mathrm{T}}, \mathrm{G}=\left(\begin{array}{llll}1 & 0 & 0 & -1\end{array}\right) / 2$

(4) $\mathrm{S}=\left(\begin{array}{llll}1 & 1 & 0 & 0\end{array}\right)^{\mathrm{T}}, \mathrm{G}=\left(\begin{array}{llll}1 & 0 & 1 & 0\end{array}\right) / 2 \square\left(\begin{array}{lll}8 \\ \mathrm{C}\end{array}\right) \mathrm{S}=\left(\begin{array}{llll}1 & -1 & 0 & 0\end{array}\right)^{\mathrm{T}}, \mathrm{G}=\left(\begin{array}{llll}1 & 0 & 1 & 0\end{array}\right) / 2$

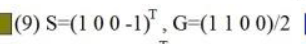

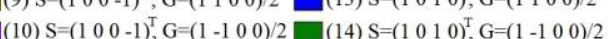

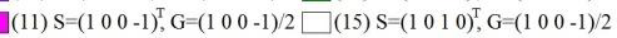

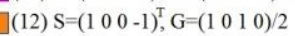

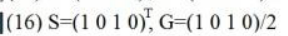

Fig. 2. Altitude profiles of the lidar signal power in the parallel $(a)$ and orthogonal $(b)$ receiving polarization channels of the lidar (March 24, 2016, 16:50-17:07)

Preprocessing of the experimental data obtained using the lidar developed at TSU includes correction for afterpulsing and erroneous signals of PMTs of the lidar receiving system and reduction of the statistical signal noise level caused by the operation regime of the PMTs in photon counting mode and subtraction of the background noise [8]. Subsequent processing of the experimental data allows determining the altitudes of the lower and upper cloud boundaries, their optical cloud thickness $\tau$, scattering ratio (determined as $R(h)=\left(\beta_{\pi}^{m}+\beta_{\pi}^{a}\right) / \beta_{\pi}^{m}$, where $h$ is the altitude, and $\beta_{\pi}^{m}$ with $\beta_{\pi}^{a}$ are the molecular and aerosol backscattering coefficients, respectively) [9], and the phase matrix of clouds at the selected altitude [10]. BSPMs determined in the experiments are normalized to the element $m_{11}$ : 


$$
\mathbf{M}_{\pi}\left(\text { March 24, 2016;11,25 km) }=\left(\begin{array}{crrr}
1 & -0,09 & 0,05 & 0,09 \\
-0,09 & 0,64 & 0,53 & -0,29 \\
-0,05 & -0,14 & -0,34 & -0,15 \\
0,09 & 0,15 & -0,09 & -0,41
\end{array}\right) .\right.
$$

A considerable array of the experimental data is accumulated over the years of operation of the lidar. However, results of processing these data are some data arrays including altitude profiles of lidar signal power, separated geometrical and optical parameters of clouds, as well as meteorological conditions of observations of clouds. At present, a unified base of experimental data combining the listed arrays, systematizing them, and providing quick access to information from each of them is developing. The arrays are extended with new data, when meteorological conditions at the place of the lidar location allow the experiments to be performed.

\section{Obtaining BSPMs of polydisperse cirrus calculated theoretically for the comparison with experimental data}

Accumulated array of the experimental data obtained using the polarization laser sensing method is statistically significant, however the problem of their interpretation related with the evaluation of the parameters of orientation of crystalline particles is still not solved. This is due to the absence of a strict theory of scattering of electromagnetic waves on an ensemble of spatially oriented ice particles. Our approach to solving the inverse problem - determining the microstructure parameters of clouds - is to compare BSPMs obtained experimentally with ones calculated theoretically. For this purpose, we use the database developed at V. E. Zuev Institute of Atmospheric Optics (IAO SB RAS; Tomsk, Russia) including BSPMs calculated for monodisperse ensembles of ice crystals for various parameters of spatial orientation [11]. The method of physical optics based on the Kirchhoff diffraction theory is used during the calculation of the elements of BSPMs [12].

To obtain BSPMs of polydisperse ensembles of hexagonal ice plates, data from the mentioned database were approximated for further comparison of them with the results of the experiments [13]. Such matrices were calculated as additive sums (with weight coefficients) of BSMPs of monodisperse ensembles taking into account the real parameters of distribution functions of sizes of the cloud particles and parameters of spatial orientation. To approximate the theoretical matrices array, the gamma function was selected $[14,15]$ :

$$
f\left(a_{0}\right)=\frac{\mu^{\mu+1}}{\Gamma(\mu+1) a_{0 m}}\left(\frac{a_{0}}{a_{0 m}}\right)^{\mu} \exp \left(-\mu \frac{a_{0}}{a_{0 m}}\right)
$$

where $a_{0}=\sqrt{h D / \pi}$ is the equivalent size of the plates, $\mu$ is the variance of the distribution, and $a_{0 m}$ is the modal equivalent size. To approximate the orientation angle distribution of the particles, Gaussian function was used $[12 ; 16]$ :

$$
f(\theta)=b \cdot \exp \left[-\left(\theta-\theta_{0}\right)^{2} / 2 \theta_{S}\right],
$$

where $\mathrm{b}$ is the normalization constant, $\theta_{0}$ is the modal angle, and $\theta_{s}$ is the variance of the distribution. Since ranges of sizes of the particles are different from those in [14] (Fig. 3), we used another values of the modal sizes. 

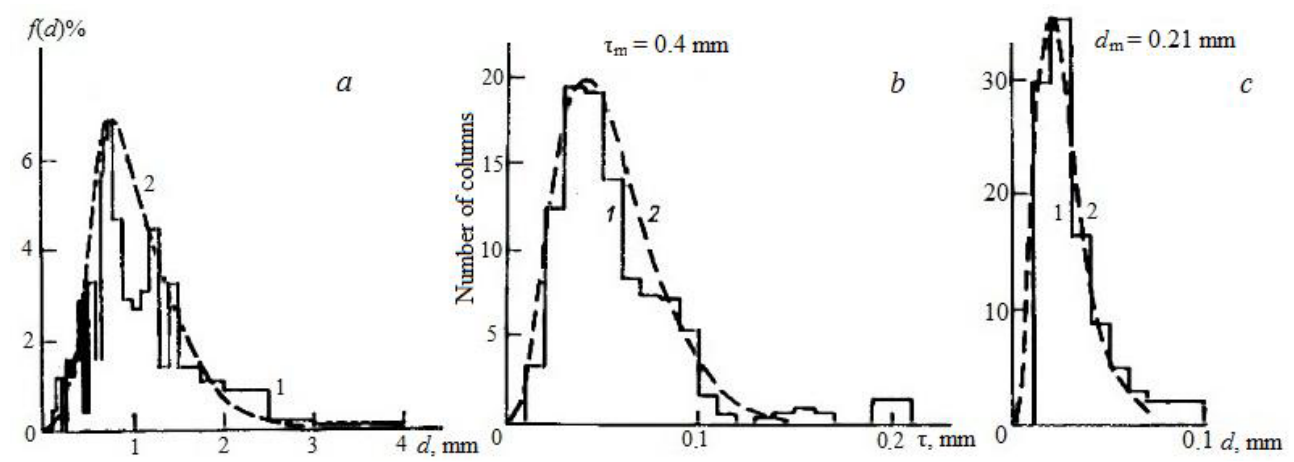

Fig. 3. Size distributions of ice plates $\left(a: d_{m}=0.8 \mathrm{~mm}, \mu=4\right)$ and columns $(b$ and $c: \mu=3)$ in cirrus: bar chart 1 is for experiment and curve 2 is for gamma distribution [14]

An extended version of the database developed at IAO SB RAS contains BSPMs calculated for regular shape hexagonal plates and columns [17]. To form the matrices for polydisperse ensembles of ice crystals consisting of the particles of both forms with the parameters of distributions of the particles corresponding to real cirrus, the relation of their fraction from the results of [18] were used. In this work, aggregated data on experimental investigations of microstructure of cirrus obtained in the framework of the projects TWPICE (tropics, 2006), ISDAC (Arctic, 2008), and SPARTICUS (middle latitudes, 2010) are shown for ice plates, columns, and crystals of other shapes. The SPARTICUS data [19] were chosen as the closest to the climatic conditions of Western Siberia. Since morphological properties of investigated clouds are not taken into account during processing the data obtained using the lidar developed at TSU, averaged percentages of the crystals were used for obtaining the BSPMs of polydisperse ensembles of aerosol particles (Table 1).

Table 1. Percentages of ice hexagonal columns and plates in cirrus normalized to the total number of regular shape ice crystals [18].

\begin{tabular}{|c|c|c|}
\hline Temperature & $-67<T<-35^{\circ} \mathrm{C}$ & $-40<T<-15^{\circ} \mathrm{C}$ \\
\hline Columns & $47.9(43.7 ; 48.7)$ & $45.4(27.66 ; 52.6)$ \\
\hline Plates & $7.9(30.6 ; 3.9)$ & $24.3(57.85 ; 10.6)$ \\
\hline
\end{tabular}

Note: values in parentheses correspond to fractions of crystals in clouds formed from anvils and in another clouds, respectively

\section{Conclusions}

Functions of distribution of particles in shape, size, and spatial orientation, as well as parameters of these functions corresponding to real high-level clouds were used for obtaining backscattering matrices of HLCs on the basis of matrices calculated theoretically. With this in mind, the described BSPMs can be considered as matrices of HLCs calculated theoretically. Formation of the array of such matrices calculated for various values of parameters of the distributions will allow the inverse problem of estimation of microstructure parameters of real clouds to be solved. Comparison of these data arrays with meteorological conditions at the altitudes of formation of HLCs will allow drawing conclusions about conditions of the formation of clouds with a particular microstructure.

This work was supported in part by the Russian Foundation for Basic Research (grant No. 16-05-00710) and the Ministry of Education and Science of the Russian Federation in the framework of the Tomsk State University Academic D.I. Mendeleev Fund Program (project No. 8.1.12.2015). 


\section{References}

1. B.V. Kaul, S.N. Volkov, and I.V. Samokhvalov, Opt. Atm. Okeana, 16, No. 4, 354 361 (2003).

2. I.V. Samokhvalov, B.V. Kaul, S.V. Nasonov, et al., Opt. Atm. Okeana, 25, No. 5, 403411 (2012).

3. M.D. Guasta, E. Vallar, O. Riviere, et al., Appl. Opt., 45, No. 20, 4878-2887 (2006).

4. M. Hayman, S. Spuler, B. Morley, et al., Opt. Exp., 20, No. 28, 29553-29567 (2012).

5. S.N. Volkov, I.V. Samokvalov, H.D. Cheong, et al., Appl. Opt., 54, No. 11, 30953105 (2015).

6. E.V. Nee and V.V. Bryukhanova, Proc. SPIE, 9680, 96804M-1-96804M-5 (2015).

7. B.V. Kaul and I.V. Samokhvalov, Atm. Ocean. Opt., 18, No. 11, 866-870 (2005).

8. S.N. Volkov, Optical characteristics of high-level clouds from the data on lidar sensing (Candidate's dissertation in physical-technical sciences, Tomsk, 1986).

9. I.V. Samokhvalov, I.D. Bryukhanov, S.V. Nasonov, et al., Russ. Phys. J., 55, No. 8, 925-929 (2013).

10. I.V. Samokhvalov, S.N. Volkov, I.D. Bryukhanov, et al., Proc. SPIE, 9680, 96804G-196804G-4 (2015).

11. ftp://ftp.iao.ru/pub/GWDT/Physical_optics.

12. A. Borovoi, A. Konoshonkin, N. Kustova, et al., Opt. Exp., 20, No. 27, 2822228233 (2012).

13. I.V. Samokhvalov, S.V. Nasonov, I.D. Bryukhanov, et al., Izv. Vyssh. Ucheb. Zaved. Fiz., 56, No. 8/3, 281-283 (2013).

14. O.A. Volkovitskii, L.N. Pavlova, and A.G. Petrushin, Optical properties of crystalline clouds (Gidrometeoizdat, Leningrad, 1984).

15. V.E. Zuev, Propagation of laser radiation in the atmosphere (Radio i svyaz', Moscow, 1981).

16. K. Sato and H. Okamoto, J. Geophys. Res., 111, D22213 (2006).

17. A.V. Konoshonkin, N.V. Kustova, V.A. Shishko, et al., Opt. Atm. Okeana, 29, No. 1, 40-50 (2016).

18. J. Um, G.M. McFarquhar, Y.P. Hong, et al., Atmos. Chem. Phys., 15, 39333956 (2015).

19. http://acrf-campaign.arm.gov/sparticus. 